\title{
Phase Transition and Optical Properties for Ultrathin $\mathrm{KNbO}_{3}$ Nanowires
}

\author{
Shulin Yang, ${ }^{1}$ Yongming Hu, ${ }^{1}$ Shengfu Wang, ${ }^{1}$ Haoshuang Gu, ${ }^{1}$ and Yu Wang ${ }^{2}$ \\ ${ }^{1}$ Hubei Collaborative Innovation Center for Advanced Organic Chemical Materials, Key Lab of Ferro- \& Piezo Materials and \\ Devices of Hubei Province, Faculty of Physics \& Electronic Technology, Hubei University, Wuhan 430062, China \\ ${ }^{2}$ Department of Applied Physics, The Hong Kong Polytechnic University, Hong Kong
}

Correspondence should be addressed to Yongming Hu; yongming.hu09@gmail.com

Received 28 October 2013; Accepted 7 November 2013

Academic Editor: Danyang Wang

Copyright (c) 2013 Shulin Yang et al. This is an open access article distributed under the Creative Commons Attribution License, which permits unrestricted use, distribution, and reproduction in any medium, provided the original work is properly cited.

Fascicular $\mathrm{KNbO}_{3}$ nanowires with tetragonal perovskite structures and ultrasmall diameters are synthesized by hydrothermal route at about $150^{\circ} \mathrm{C}$ for 24 hours. The concentrations of medium alkalinity have influenced phase structures and the final morphologies of the products significantly by modifying the conditions in process. The as-prepared $\mathrm{KNbO}_{3}$ nanowires exhibit three phase transitions at about $343,454.7$, and $623 \mathrm{~K}$ as the temperature increases from 250 to $700 \mathrm{~K}$. The band gap is about $3.78 \mathrm{eV}$ for $\mathrm{KNbO}_{3}$ nanowires. Photoluminescence study at room temperature reveals two visible light emission bands peaking at $\sim 551$ and $597 \mathrm{~nm}$, respectively, which may be due to the oxygen vacancies, site niobium (occupy the location of $\mathrm{Nb}$ ), and antisite niobium (occupy the location of $\mathrm{K})$ in $\mathrm{KNbO}_{3}$ nanowires.

\section{Introduction}

Perovskite-type metallic oxides make a family of materials that share a common crystal structure, analogy to that of the natural crystal calcium titanate, yet exhibit a broad range of interesting physical properties [1]. With the advances in science and technology of integrated ferroelectrics, the size-dependent ferroelectric behaviors become crucial due to the dimensional confinement or the size of ferroelectrics decreasing [2]. 1D nanostructure of ferroelectric materials, such as nanorods, nanowires, and nanotubes, have attracted great attention due to their promising applications, such as miniaturized piezoelectric actuators, medical imaging detectors, and even ferroelectric memories [3, 4]. In recent years, the size-dependent physical properties, such as phase transition, polarizations, and energy band, in ferroelectric nanowires have been evaluated systematically. For instance, Naumov and Fu revealed that both the Curie temperature and the polarization of $\mathrm{Pb}\left(\mathrm{Zr}_{0.5} \mathrm{Ti}_{0.5}\right) \mathrm{O}_{3}$ nanowire decreased with the reducing diameter [5]. Spanier et al. reported that phase transition temperature of $\mathrm{BaTiO}_{3}$ nanowires fell below room temperature as the diameter was $\sim 3 \mathrm{~nm}$ [6]. Geneste et al. found that the ferroelectric properties of $\mathrm{BaTiO}_{3}$ nanowire vanished when the diameter was less than $1.2 \mathrm{~nm}$ according to the first principle calculation [7]. However, Morozovska et al. reported that the Curie temperature of ferroelectric nanorods/nanowires could be higher than that of bulk materials from a direct variational method [8]. In addition, the author revealed that there was a blue-green emission for $\mathrm{PbTiO}_{3}$ nanowires (with diameters of $10 \sim 20 \mathrm{~nm}$ ) both the oxygen vacancies and lead-oxygen vacancy centers may be responsible for this phenomenon [9]. Despite very promising progress in theory, there is inconsistence for phase transition as the diameter of ferroelectric nanowires is decreased. Moreover, few reports have concerned the investigation of the phase transition behavior in experiments.

Potassium niobate $\left(\mathrm{KNbO}_{3}\right)$ is one of the most important ferroelectric perovskite oxides, which undergoes successive phase transitions upon cooling from its cubic phase, that is, from cubic to tetragonal at $435^{\circ} \mathrm{C}$, tetragonal to orthorhombic at $225^{\circ} \mathrm{C}$, and orthorhombic to rhombohedral at $-10^{\circ} \mathrm{C}[10$, 11]. This material attracts great interests for its multiple 
applications, such as optical waveguides, frequency doublers, holographic storage systems, and other technological devices [12-14]. The great potential has stimulated research on the $\mathrm{KNbO}_{3}$ nanowires. Therefore, the synthesis, phase transition, and optical properties of $\mathrm{KNbO}_{3}$ nanowires are worth being investigated. Magrez et al. realized the growth of $\mathrm{KNbO}_{3}$ nanowires with a narrow diameter distribution $\sim 60 \mathrm{~nm}$ via a hydrothermal route at $150^{\circ} \mathrm{C}$ from a $38.7 \mathrm{wt} \%$ $\mathrm{KOH}, 0.4 \mathrm{wt} \% \mathrm{Nb}_{2} \mathrm{O}_{5}$, and $60.9 \mathrm{wt} \% \mathrm{H}_{2} \mathrm{O}$ mixture [15]. Baier-Saip et al. reported the influence of grain size on the phase transition of ferroelectric $\mathrm{KNbO}_{3}$ [16]. Wang et al. found that the phase transition of orthorhombic to tetragonal and tetragonal to cubic of $\mathrm{KNbO}_{3}$ nanorods with diameters of $100 \sim 300 \mathrm{~nm}$ occurred at lower temperatures than that of bulk counterpart due to finite size or disorder effects [17]. Nakayama et al. developed an electrode-free, continuously tunable coherent visible light source compatible with physiological environments from individual $\mathrm{KNbO}_{3}$ nanowires, exhibiting efficient second harmonic generation, which could act as frequency converters, allowing the local synthesis of a wide range of colors via sum and difference frequency generations [18]. However, the diameters of the $1 \mathrm{D}$ nanostructure synthesized by most of the current routes are much larger than the critical size in ferroelectrics. It is still very challenging to obtain ferroelectric oxide nanowires with extremely small diameters $(<10 \mathrm{~nm})$ and a narrow size distribution, especially for niobate oxide. At the same time, the size effects and surface states have important impacts on the lattice distortion in the crystal, which will further result in the changes of the phase transition temperature. Thus, finding a feasible way to overcome those drawbacks discussed ahead is very essential. Herein, $\mathrm{KNbO}_{3}$ nanowires with ultrathin diameters (down to $5 \mathrm{~nm}$ ) are synthesized through two step method, that is, milling of raw materials followed by hydrothermal treatment at $150^{\circ} \mathrm{C}$ for $12 \mathrm{~h}$. In addition, in the phase transition, room temperature photoluminescence properties of $\mathrm{KNbO}_{3}$ nanowires are also investigated. The studies show that the $\mathrm{KNbO}_{3}$ nanowires may be a promising candidate for fabricating piezoelectric sensors and other functional devices.

\section{Experiments}

2.1. Synthesis of $\mathrm{KNbO}_{3}$ Nanowires. Niobium pentoxide $\left(\mathrm{Nb}_{2} \mathrm{O}_{5}, 99.95 \%\right)$ and potassium hydroxide $(\mathrm{KOH}, 95 \%)$ were used as raw materials and $\mathrm{C}_{12} \mathrm{H}_{25} \mathrm{OH}$ as surfactant. Figure 1 shows the process illustration of $\mathrm{KNbO}_{3}$ nanowires through two step hydrothermal method. Firstly, $\mathrm{Nb}_{2} \mathrm{O}_{5}$ powders of $13.29 \mathrm{~g}, \mathrm{KOH}$ of $5.89 \mathrm{~g}$, and $\mathrm{C}_{12} \mathrm{H}_{25} \mathrm{OH}$ of $2 \mathrm{~g}$ were dispersed in $80 \mathrm{~mL} \mathrm{C}_{2} \mathrm{H}_{5} \mathrm{OH}$ together. The above mixture was milled at room temperature for 12 hours. Then the obtained precursor was dried by infrared lamp for 12 hours. After that, $20 \%$ amount of the above precursor, $20 \mathrm{~mL} \mathrm{C}_{2} \mathrm{H}_{5} \mathrm{OH}$, and $20 \mathrm{~mL} \mathrm{H}_{2} \mathrm{O}$ were transferred into the stainless steel autoclave $(50 \mathrm{~mL})$. The next step is to add $1.2 \mathrm{~g} \mathrm{KOH}$ in the above mixture and stir for $30 \mathrm{~min}$. Finally, $\mathrm{KNbO}_{3}$ nanowires were synthesized after the mix was subjected to further hydrothermal treatment at $150^{\circ} \mathrm{C}$ for $12 \mathrm{~h}$ and cooled to room temperature.

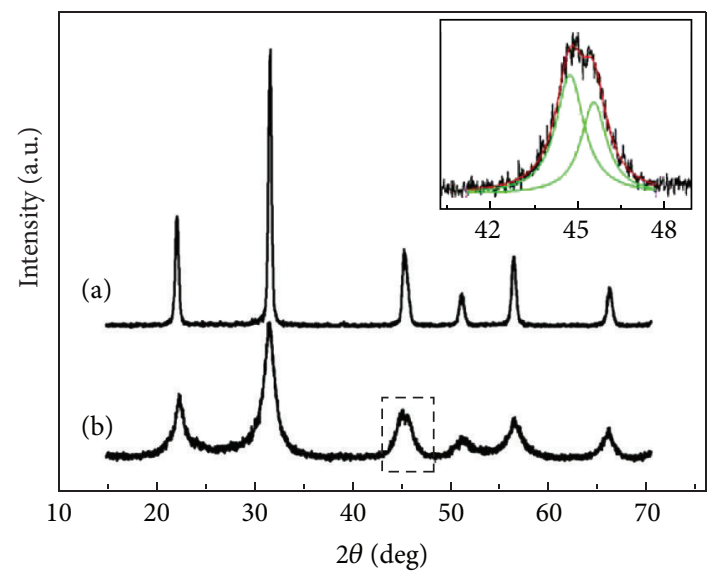

FIGURE 1: XRD patterns of (a) nanoparticles and (b) nanowires of $\mathrm{KNbO}_{3}$. Inset: the Lorentz fitting for diffraction peak of $\mathrm{KNbO}_{3}$ nanowires located at $2 \theta$ of $\sim 45^{\circ}$.

2.2. Characterization of Materials. X-ray diffraction (XRD) measurements were performed on a Bruker AXS-D8Advance X-ray Diffractometer system using $\mathrm{CuK} \alpha(\lambda=$ $1.5406 \AA$ ) as the radiation source. Microstructures of the samples were determined on a field-emission scanning electron microscopy (FESEM) JEOL JSM-6700F and transmission electron microscopy (TEM) JEOL 2011 using an acceleration voltage of $200 \mathrm{kV}$. The phase transition properties of $\mathrm{KNbO}_{3}$ nanowires were measured using a differential scanning calorimeter (DSC, Perkin-Elmer) in the range of 250 to $700 \mathrm{~K}$ at a heating and cooling rate of $10.0 \mathrm{~K} / \mathrm{min}$ in a nitrogen atmosphere. The UV-vis absorption spectra were measured using Shimadzu UV-2550 spectrophotometer. The fluorescence spectrum of $\mathrm{KNbO}_{3}$ nanowires was recorded with Edinburgh FLSP920 fluorimeter. Fluorescence intensities were corrected for inner filter effects according to standard methods.

\section{Results and Discussions}

We have conducted a series of experiments with various concentrations of medium alkalinity, which have significant effects on the phase structures and the final morphologies of the products. We find that $\mathrm{KNbO}_{3}$ nanoparticles were obtained when the total weight of $\mathrm{NaOH}$ reached up to 15 grams, while $\mathrm{KNbO}_{3}$ nanowires were synthesized as the weight was 7.09 grams during hydrothermal treatment. Figure 1 shows the XRD pattern of $\mathrm{KNbO}_{3}$ particles (a) and nanowires (b) synthesized by means of hydrothermal method, respectively. One can see that the sharp diffraction peak indicated that $\mathrm{KNbO}_{3}$ particles have better crystallization than nanowires. All the diffraction peaks of them are well consistent with that of Joint Committee on Powder Diffraction Standards (JCPDS) Cards no. 71-0946 and 710946, respectively, illustrating clearly that the samples are composed of $\mathrm{KNbO}_{3}$ perovskite structure and there is no trace of any impurity phase in the samples. It is noted that $\mathrm{KNbO}_{3}$ nanoparticles have the orthorhombic structure and $\mathrm{KNbO}_{3}$ nanowires belong to the tetragonal structure, which 


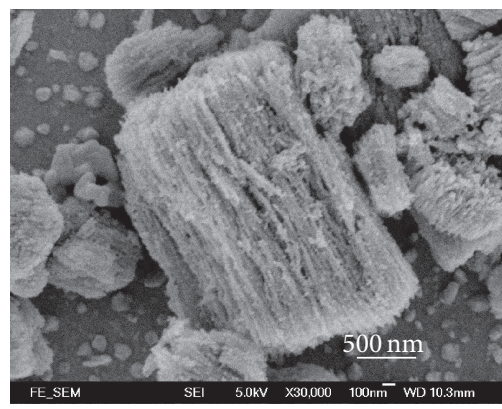

(a)

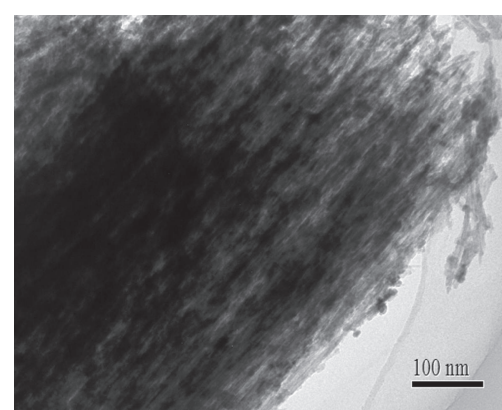

(b)

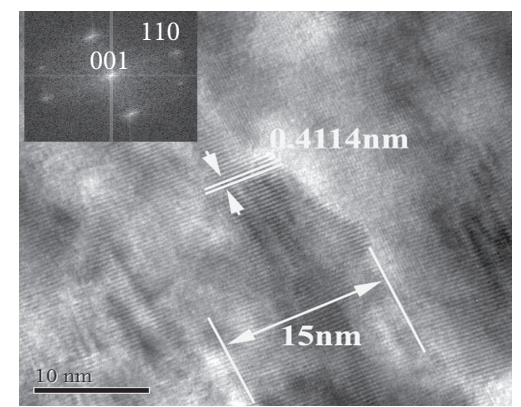

(c)

FIgURE 2: (a) SEM image, (b) low magnification TEM image, and (c) HRTEM image of the $\mathrm{KNbO}_{3}$ nanowires. Inset: SAED pattern of individual nanowires.

can be identified by the splitting peak located at $2 \theta \sim 45^{\circ}$ (shown in the inset in Figure 1). The refined lattice parameters obtained by Curve Fitting software are determined to be $a$ $=4.0124(3)$ and $c=4.0456(5) \AA$ for $\mathrm{KNbO}_{3}$ nanowires, while that parameters for nanoparticles are $a=3.9966(6), b=$ 5.6535(5), and $c=5.6577(6) \AA$ for nanoparticles. At the same time, the strain in the samples has been studied. The consequences imply that the strain in $\mathrm{KNbO}_{3}$ nanowires is about $33 \pm 2 \%$, while it is about $10 \pm 3 \%$ for $\mathrm{KNbO}_{3}$ nanoparticles.

The typical SEM and TEM images of the $\mathrm{KNbO}_{3}$ nanowires are shown in Figure 2. As can be seen in Figure 2(a), the parallel nanowires are arranged side by side and tend to form the fascicular structure due to the Van der Waals and/or electrostatic attraction force. Almost all of the nanowires grow into regular structure and distribute uniformly in both diameter and length. As demonstrated in Figure 2(b), the TEM image exhibits a large quantity of nanowires with diameters of $5 \sim 15 \mathrm{~nm}$ and length of up to several micrometers. The HRTEM image in Figure 2(c) shows the lattice fringes of the sample, indicating that the $\mathrm{KNbO}_{3}$ nanowires are well crystallized and structurally uniform. The adjacent lattice spacing is about $0.4114 \mathrm{~nm}$, corresponding to the (001) lattice plane of $\mathrm{KNbO}_{3}$. The SAED pattern (inset of Figure 2(c)), which is indexed by assuming the lattice constants refined from XRD measurements, is obtained from an entire nanowire. The result shows clear diffraction spots characteristic of crystalline $\mathrm{KNbO}_{3}$, demonstrating these nanowires are growing along [001] directions.

It is well known that the heat energy released from oxide materials when the phase transition occurs. Thus, differential scanning calorimeter (DSC) technique was selected to reveal the phase transition behavior of $\mathrm{KNbO}_{3}$ nanowires with diameters of $5 \sim 15 \mathrm{~nm}$. Figure 3 shows the DSC curve for $\mathrm{KNbO}_{3}$ nanowires recorded in the temperature range from 250 to $700 \mathrm{~K}$. It can be seen that the as-prepared nanowires go through three phase transitions at about $623,454.7$, and $343 \mathrm{~K}$, respectively, as the temperature cools from 700 to $250 \mathrm{~K}$. According to the studies in the literature, $\mathrm{KNbO}_{3}$ crystal undergoes successive ferroelectric transitions upon cooling from its cubic $\mathrm{ABO}_{3}$ perovskite phase [9]. Therefore, it can be concluded that the structure will transform from cubic to tetragonal when the sample is cooled to $623 \mathrm{~K}$; it

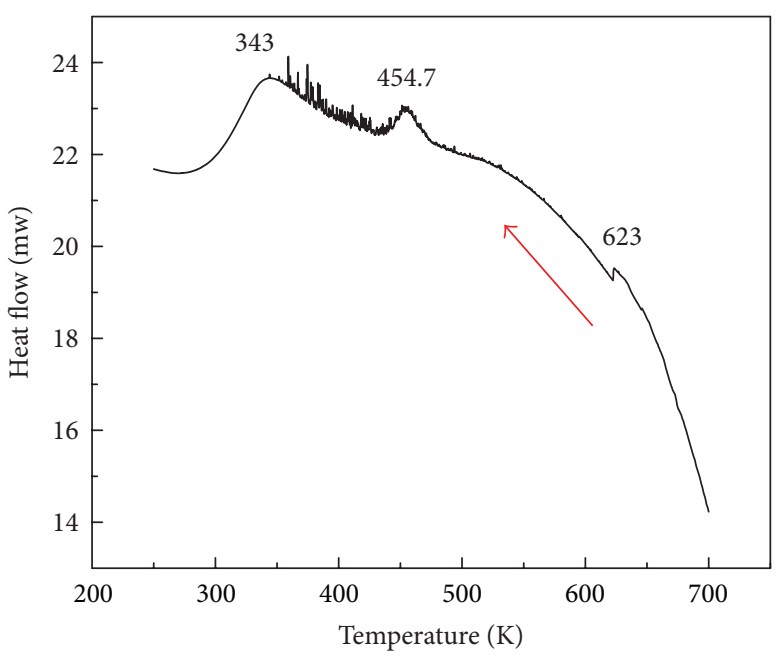

FIGURE 3: DSC curve for $\mathrm{KNbO}_{3}$ nanowires recorded at the temperature range from 250 to $700 \mathrm{~K}$.

will change to orthorhombic structure as the temperature reaches $\sim 454.7 \mathrm{~K}$, and it shows the rhombohedra structure when the sample is below $\sim 343 \mathrm{~K}$. However, Gopalan and Raj reported that the phase of the epitaxial $\mathrm{KNbO}_{3}$ thin films would transform to be $210 \pm 10^{\circ} \mathrm{C}$ for orthorhombictetragonal and $450 \pm 10^{\circ} \mathrm{C}$ for tetragonal-cubic transitions, which was studied by in situ second harmonic generation measurements. It can be clearly seen that the phase transition temperature of $\mathrm{KNbO}_{3}$ nanowires is much higher than that of epitaxial $\mathrm{KNbO}_{3}$ thin films [19]. We consider that the obvious size and surface effect of $\mathrm{KNbO}_{3}$ nanowires would have significant influences on the lattice distortion and phase transition, which may be also one of the important reasons for the differences discussed ahead.

In the earlier studies, we have found that the morphology of $\mathrm{KNbO}_{3}$ nanostructures has considerable influences on the onsets of absorption. Figure 4 shows the UV-vis absorption spectrum of $\mathrm{KNbO}_{3}$ nanowires dispersed in ethanol. It is clear that strong absorption happens as the wavelength is lower than $330 \mathrm{~nm}$, while there is no obvious absorption band in the whole wavelength range (190 900 nm). The band gap of $\mathrm{KNbO}_{3}$ nanowires is estimated to be $3.78 \mathrm{eV}$ from the 


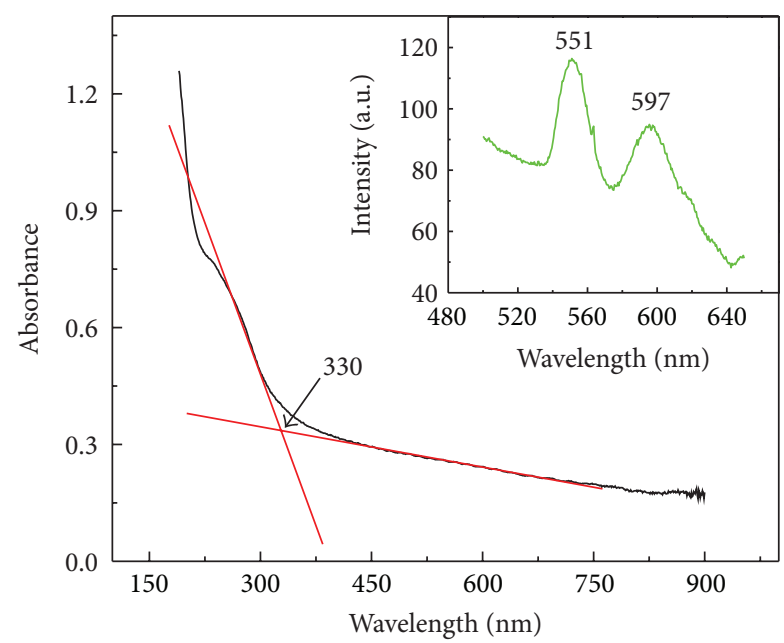

FIGURE 4: UV-vis absorption spectrum of $\mathrm{KNbO}_{3}$ nanowires, the inset is the room temperature PL spectrum.

onsets of absorption, which is higher than that of $\mathrm{KNbO}_{3}$ nanocubes with edge sizes of $\sim 80 \mathrm{~nm}(\sim 3.14 \mathrm{eV})$ [20]. The combined action of size and surface effect will result in the obvious blue shift of optical absorption or larger energy gap. The inset in Figure 4 exhibits the room temperature $\mathrm{PL}$ spectra of $\mathrm{KNbO}_{3}$ nanowires excited at wavelengths of $400 \mathrm{~nm}$. A green light emission band located at about $551 \mathrm{~nm}$ $(2.25 \mathrm{eV})$ and a yellow light located at $597 \mathrm{~nm}(2.077 \mathrm{eV})$ were observed. As mentioned above, the energy gap of $\mathrm{KNbO}_{3}$ nanowires is about $3.78 \mathrm{eV}$, which is higher than the excitation energy used in the present work. Therefore, the light emissions observed from 500 to $650 \mathrm{~nm}$ are not caused by a direct electron transition between the valence bands to the conduction bands. Similar results have also been found in $\mathrm{PbTiO}_{3}$ nanowires [8]. It has been reported that oxygen vacancies in oxides would enhance the emission intensities and extend the radiative lifetime of self-trapped excitons. As it is well known, the smaller the diameters of nanowires, the higher the surface to volume ratio, and there is more concentration of surface oxygen vacancies. Fischer et al. have reported that the luminescence of $\mathrm{LiNbO}_{3}$ can be ascribed to the recombination of the electrons captured by $\mathrm{Nb}^{5+}$ ions and holes captured by oxygen in an atomistic model: $\mathrm{Nb}^{4+}+\mathrm{O}^{-}=$ $\mathrm{Nb}^{5+}+\mathrm{O}^{2-}$, and both the site niobium (occupy the location of $\mathrm{Nb}$ ) and antisite niobium (occupy the location of $\mathrm{Li}$ ) are responsible for the main emission in $\mathrm{LiNbO}_{3}$ [21]. Therefore, the emission bands centered at wavelengths of about 551 and $597 \mathrm{~nm}$ would derive from the oxygen vacancies and the site niobium (occupy the location of $\mathrm{Nb}$ ) and antisite niobium (occupy the location of $\mathrm{K}$ ), respectively.

\section{Conclusions}

In conclusion, fascicular $\mathrm{KNbO}_{3}$ nanowires with perovskite structures and ultrathin diameters of $5 \sim 15 \mathrm{~nm}$ were synthesized by hydrothermal treatment at low temperature. The $\mathrm{KNbO}_{3}$ nanowires underwent successive phase transitions upon cooling from its cubic phase, that is, cubic $\rightarrow$ tetragonal at $623 \mathrm{~K}$, tetragonal $\rightarrow$ orthorhombic at $\sim 454.7 \mathrm{~K}$, and orthorhombic $\rightarrow$ rhombohedral at $\sim 343 \mathrm{~K}$. The band gap of $\mathrm{KNbO}_{3}$ nanowires is estimated to be $3.78 \mathrm{eV}$, which may be caused by the combined action of size effect and quantum size effect. There are two visible light emission peaks located at $\sim 551 \mathrm{~nm}(2.25 \mathrm{eV})$ and $597 \mathrm{~nm}(2.077 \mathrm{eV})$ for $\mathrm{KNbO}_{3}$ nanowires at room temperature. The oxygen vacancy, the site niobium (occupy the location of $\mathrm{Nb}$ ), and antisite niobium (occupy the location of $\mathrm{K}$ ) may have a response for these two emissions.

\section{Acknowledgments}

This work was financially supported by the National Science Foundation of China (NSFC) (Grant no. 50902046, and 61274073) and the National High Technology Research and Development Program of China (863 Program) (no. 2013AA031903), and the Youth Chenguang Project of Science and Technology, the Key Project of Science and Technology of Wuhan City, China (Grant no. 201150431133, 201160723217) and the Research Grants Council of Hong Kong (GRF no. PolyU 5309/08E).

\section{References}

[1] Y.-Z. Wang, H. Zhong, X.-M. Li et al., "Electrochemiluminsecence study of perovskite-type oxides $\mathrm{LaTiO}_{3}-\mathrm{Ag} 0.1$ with $\mathrm{Ru}(\mathrm{bpy})_{3}{ }^{2+}$ modified electrode," International Journal of Electrochemcal Science, vol. 8, pp. 1911-1919, 2013.

[2] R. Grange, J.-W. Choi, C.-L. Hsieh et al., "Lithium niobate nanowires synthesis, optical properties, and manipulation," Applied Physics Letters, vol. 95, Article ID 143105, 3 pages, 2009.

[3] J. Hong and D. Fang, "Size-dependent ferroelectric behaviors of $\mathrm{BaTiO}_{3}$ nanowires," Applied Physics Letters, vol. 92, Article ID 012906, 3 pages, 2008.

[4] Y. Li, X.-Y. Yang, Y. Feng, Z.-Y. Yuan, and B.-L. Su, "Onedimensional metal oxide nanotubes, nanowires, nanoribbons, and nanorods: synthesis, characterizations, properties and application," Critical Reviews in Solid State and Materials Sciences, vol. 37, no. 1, pp. 1-74, 2012.

[5] I. I. Naumov and H. X. Fu, "Spontaneous polarization in onedimensional $\mathrm{Pb}(\mathrm{ZrTi}) \mathrm{O}_{3}$ nanowires," Physical Review Letters, vol. 95, no. 24, Article ID 247602, 4 pages, 2005.

[6] J. E. Spanier, A. M. Kolpak, J. J. Urban et al., "Ferroelectric phase transition in individual single-crystalline $\mathrm{BaTiO}_{3}$ nanowires," Nano Letters, vol. 6, no. 4, pp. 735-739, 2006.

[7] G. Geneste, E. Bousquet, J. Junquera, and P. Ghosez, "Finitesize effects in $\mathrm{BaTiO}_{3}$ nanowires," Applied Physics Letters, vol. 88, Article ID 112906, 2006.

[8] A. N. Morozovska, E. A. Eliseev, and M. D. Glinchuk, "Ferroelectricity enhancement in confined nanorods: direct variational method," Physical Review B, vol. 73, no. 21, Article ID 214106, 13 pages, 2006.

[9] Y. Hu, H. Gu, X. Sun, J. You, and J. Wang, "Photoluminescence and Raman scattering studies on $\mathrm{PbTiO}_{3}$ nanowires fabricated by hydrothermal method at low temperature," Applied Physics Letters, vol. 88, Article ID 193120, 2006.

[10] C.-H. Wen, S.-Y. Chu, Y.-D. Juang, and C.-K. Wen, "New phase transition of erbium-doped $\mathrm{KNbO}_{3}$ polycrystalline," Journal of Crystal Growth, vol. 280, no. 1-2, pp. 179-184, 2005. 
[11] Y. Lin, X. Chen, S. W. Liu et al., "Epitaxial nature and anisotropic dielectric properties of $(\mathrm{Pb}, \mathrm{Sr}) \mathrm{TiO}_{3}$ thin films on $\mathrm{NdGaO}_{3}$ substrates," Applied Physics Letters, vol. 86, Article ID 142902, 2005.

[12] L. Goldberg, L. Busse, and D. Mehusy, "Blue light generation by frequency doubling of AlGaAs broad area amplifier emission," Applied Physics Letters, vol. 60, no. 9, pp. 1037-1039, 1992.

[13] J. -S. Lee, Y. Li, Y. Lin, S. Y. Lee, and Q. X. Jia, "Hydrogeninduced degradation in epitaxial and polycrystalline (Ba,Sr) $\mathrm{TiO}_{3}$ thin films," Applied Physics Letters, vol. 84, no. 19, pp. 3825-3827, 2004.

[14] Q. X. Jia, B. Maiorov, H. Wang et al., "Comparative study of $\mathrm{REBa}_{2} \mathrm{Cu}_{3} \mathrm{O}_{7}$ films for coated conductors," IEEE Transactions on Applied Superconductivity, vol. 15, no. 2, pp. 2723-2726, 2005.

[15] A. Magrez, E. Vasco, J. W. Seo, C. Dicker, N. Setter, and L. Forró, "Growth of single-crystalline $\mathrm{KNbO}_{3}$ nanostructures," Journal of Physical Chemistry B, vol. 110, no. 1, pp. 58-61, 2006.

[16] J. A. Baier-Saip, E. Ramos-Moor, and A. L. Cabrera, "Raman study of phase transitions in $\mathrm{KNbO}_{3}$," Solid State Communications, vol. 135, no. 6, pp. 367-372, 2005.

[17] G. Z. Wang, S. M. Selbach, Y. Yu, X. Zhang, T. Grande, and M.A. Einarsrud, "Hydrothermal synthesis and characterization of $\mathrm{KNbO}_{3}$ nanorods," CrystEngComm, vol. 11, no. 9, pp. 1958-1963, 2009.

[18] Y. Nakayama, P. J. Pauzauskie, A. Radenovic et al., "Tunable nanowire nonlinear optical probe," Nature, vol. 447, pp. 10981101, 2007.

[19] V. Gopalan and R. Raj, "Domain structure and phase transitions in epitaxial $\mathrm{KNbO}_{3}$ thin films studied by in situ second harmonic generation measurements," Applied Physics Letters, vol. 68, no. 10, pp. 1323-1325, 1996.

[20] J. W. Liu, G. Chen, Z. H. Li, and Z. G. Zhang, "Hydrothermal synthesis and photocatalytic properties of $\mathrm{ATaO}_{3}$ and $\mathrm{ANbO}_{3}$ $(\mathrm{A}=\mathrm{Na}$ and $\mathrm{K})$," International Journal of Hydrogen Energy, vol. 32, no. 13, pp. 2269-2272, 2007.

[21] C. Fischer, M. Wohlecke, T. Volk, and N. Rubinina, "Influence of the damage resistant impurities $\mathrm{Zn}$ and $\mathrm{Mg}$ on the UV-excited iuminescence in $\mathrm{LiNbO}_{3}$," Physica Status Solidi A, vol. 137, no. 1, pp. 247-255, 1993. 

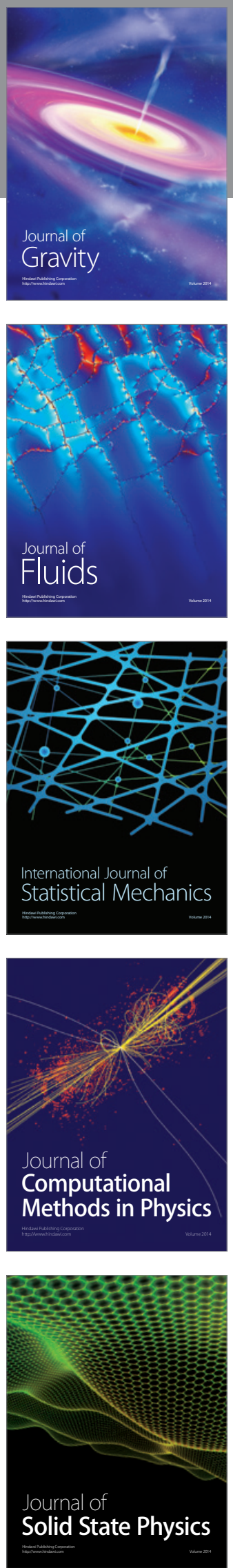

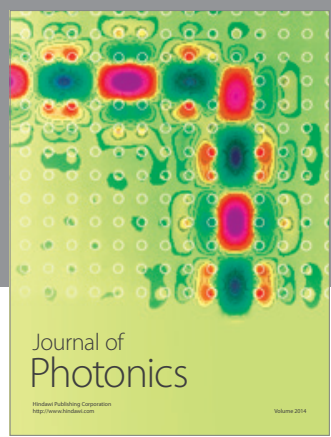

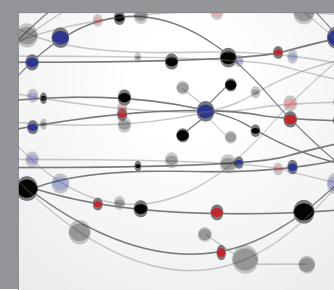

The Scientific World Journal

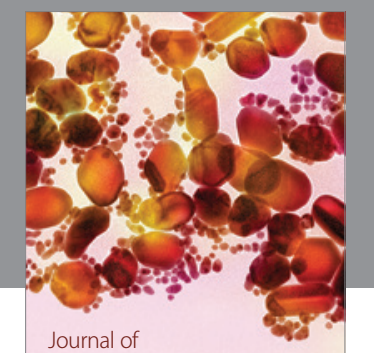

Soft Matter
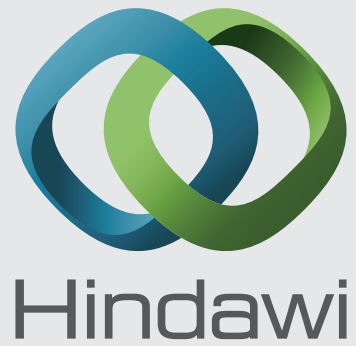

Submit your manuscripts at

http://www.hindawi.com
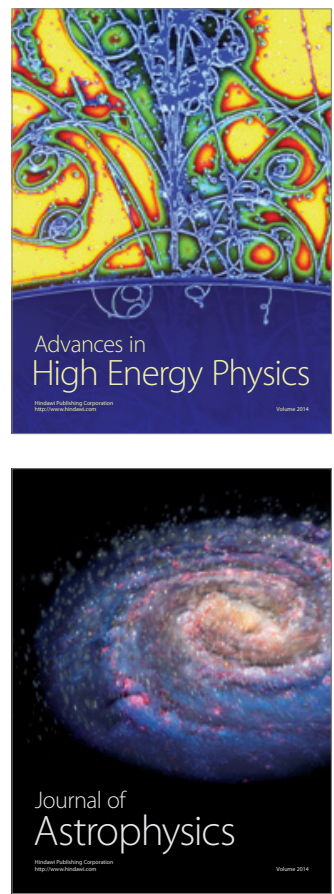
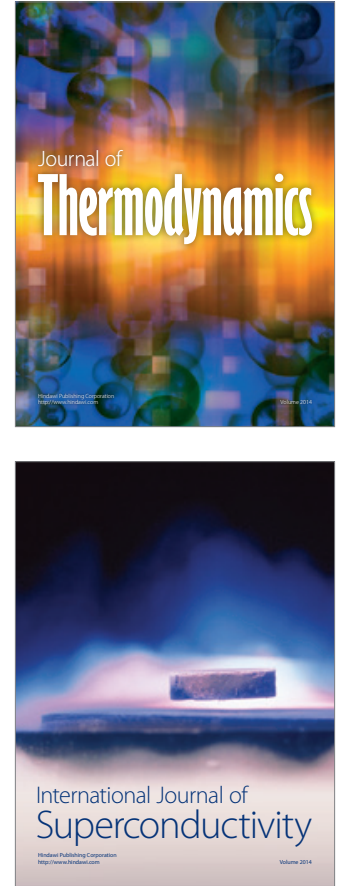
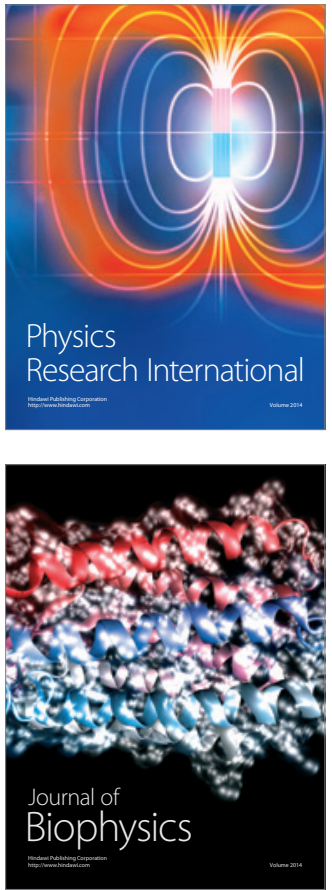
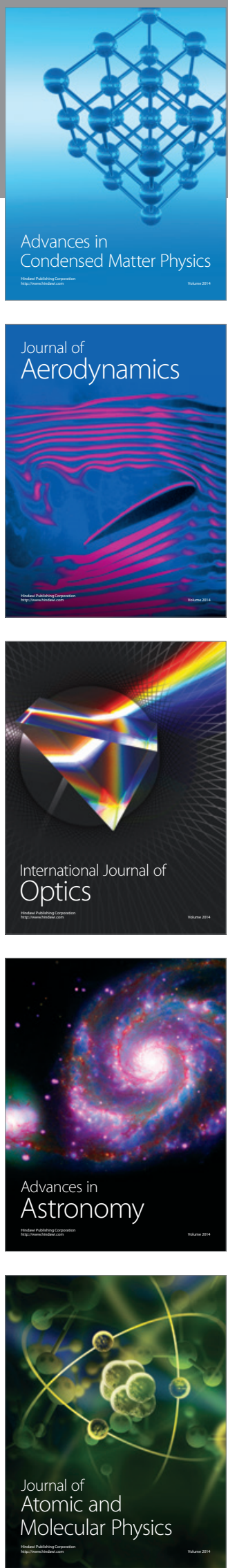